\section{Lymphogranuloma venereum presenting as genital ulceration and inguinal syndrome}

To THE EDITOR: Diagnoses of lymphogranuloma venereum (LGV; caused by the L1-L3 serovars of Chlamydia trachomatis) worldwide have increased dramatically among men who have sex with men over the past 8 years. In New South Wales, 35 cases were diagnosed in 2011, compared with 2-3 per year before 2008. ${ }^{1}$

Traditionally, LGV is described as causing a primary ulcerative genital lesion 3-30 days after exposure, leading to a secondary inguinal syndrome of buboes, which may rupture, and constitutional symptoms. ${ }^{2}$ However, this appears to be uncommon among current cases; the overwhelming majority of patients present with proctitis. ${ }^{3}$ Untreated, LGV can lead to genital scarring and anorectal strictures.

Recently, a 37-year-old HIVpositive man with a history of treated syphilis presented to our clinic with a 1-week history of a small painless indurated penile ulcer. He reported unprotected insertive anal sex 2 weeks previously with an HIV-positive casual male partner.

Tests for herpes simplex virus and polymerase chain reaction (PCR) testing for Treponema pallidum were negative. Urine testing and rectal and pharyngeal swabs were negative for chlamydia and a PCR test for gonorrhoea was also negative. A rapid plasma reagin titre for syphilis was non-reactive (negative). The patient

Painful erythematous inguinal lumps that appeared 1 month after the patient presented with a small, painless penile ulcer

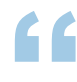

non-epidemic strains of

V. cholerae are associated with bacteraemia and a poor prognosis

Hsu et al was reviewed 1 week later and the ulcer had healed.

One month later, the patient re-presented with a 4-day history of extremely painful erythematous inguinal lumps (Box), accompanied by fever. An abdominal computed tomography scan showed multiple enlarged bilateral inguinal lymph nodes, which were aspirated. Bacterial, mycobacterial and fungal cultures of the aspirate were negative; cytological examination of the aspirate showed suppurative lymphadenitis.

Testing of the node aspirate for C. trachomatis was positive by strand displacement amplification and confirmed as serovar L2b (an LGVassociated serovar), and the patient was commenced on 3 weeks of twice daily oral doxycycline in line with a diagnosis of LGV. Retrospective testing of the original ulcer sample was also positive for serovar L2b, confirming the primary and secondary course of LGV infection in this case.

The current resurgence of LGV has drawn most attention to its potential for causing proctitis; however, this case highlights the need for awareness of LGV as a cause of genital ulceration in a high-risk population. It is not known why cases of secondary proctitis among men who have sex with men far outnumber those of classic primary ulcerative LGV or inguinal syndrome. It may be that routes of transmission exist within this population that do not require peno-anal contact, that the $\mathrm{L} 2 \mathrm{~b}$ serovar has a predisposition for rectal infection, or that primary lesions associated with LGV are far less common than previously thought. LGV has been detected in the oropharynx, but the contribution 
of this to genital and anal infection is not known. ${ }^{4}$

The ulceration associated with LGV is transient and often overlooked, and chlamydia is infrequently found elsewhere during standard sexually transmissible infection screening of the urethra, rectum or cervix in these patients. ${ }^{5}$ Thus, unless there is a high index of suspicion, an opportunity to consider the diagnosis may not arise until buboes develop. At this point it is likely that the patient will present to a general practitioner, haematologist, surgeon or urologist and that a sexually transmitted cause may be just one of many differential diagnoses.

Phillip J Read Sexual Health Physician' Anna McNulty Sexual Health Physician²

1 Kirketon Road Centre, Sydney, NSW.

2 Sydney Sexual Health Centre, Sydney, NSW. phillip.read@sesiahs.health.nsw.gov.au

Acknowledgements: We thank Alex Matthews, Prince of Wales Hospital, for lymph node aspiration, and staff of the Clinical Virology laboratory, Institute of Clinical Pathology and Medical Research, Westmead Hospital, for LGV identification.

Competing interests: No relevant disclosures.

doi: 10.5694/mjal3.10194

1 NSW Health. Lymphogranuloma venerueum (LGV) notifications in NSW residents. Communicable Diseases Branch and Centre for Epidemiology and Evidence. http://www.health.nsw.gov. au/data/diseases/lgv.asp (accessed Jan 2013).

2 White J. Manifestations and management of lymphogranuloma venereum. Curr Opin Infect Dis 2009; 22: 57-66.

3 deVriezeN, van Rooijen M, van der Loeff M,

De Vries H. Anorectal and inguinal

lymphogranuloma venereum among men who

have sex with men in Amsterdam, the

Netherlands: trends over time, symptomatology

and concurrent infections. Sex Transm Infect

2013; Feb 20 [Epub ahead of print]. doi: 10.1136/ sextrans-2012-050915.

4 Dosekun O, Edmonds S, Stockwell S, et al. Pharyngeal lymphogranuloma venereum: four cases in men who have sex with men. Sex Transm Infect 2012; 88 Suppl 1: A9. doi: 10.1136/sextrans2012-050601b.1.
5 Sethi G, Allason-Jones E, Richens J, et al. Lymphogranuloma venereum presenting as genital ulceration and inguinal syndrome in men who have sex with men in London, United Kingdom. Sex Transm Infect 2009; 85: 165-170. 